\title{
Uplink Capacity of 802.16j Mobile Multihop Relay Networks with Transparent Relays
}

\author{
Wang, Hua; Andrews, Jeffrey G.; Iversen, Villy Bæk
}

Published in:

Global Telecommunications Conference, 2009. GLOBECOM 2009. IEEE

Link to article, DOI:

10.1109/GLOCOM.2009.5425418

Publication date:

2009

Document Version

Publisher's PDF, also known as Version of record

Link back to DTU Orbit

Citation (APA):

Wang, H., Andrews, J. G., \& Iversen, V. B. (2009). Uplink Capacity of 802.16j Mobile Multihop Relay Networks with Transparent Relays. In Global Telecommunications Conference, 2009. GLOBECOM 2009. IEEE (pp. 1-6). IEEE. https://doi.org/10.1109/GLOCOM.2009.5425418

\section{General rights}

Copyright and moral rights for the publications made accessible in the public portal are retained by the authors and/or other copyright owners and it is a condition of accessing publications that users recognise and abide by the legal requirements associated with these rights.

- Users may download and print one copy of any publication from the public portal for the purpose of private study or research.

- You may not further distribute the material or use it for any profit-making activity or commercial gain

- You may freely distribute the URL identifying the publication in the public portal 


\title{
Uplink Capacity of 802.16j Mobile Multihop Relay Networks with Transparent Relays
}

\author{
Hua Wang ${ }^{\dagger}$, Jeffrey G. Andrews ${ }^{\diamond}$, and Villy B. Iversen ${ }^{\dagger}$ \\ ${ }^{\dagger}$ Department of Communications, Optics \& Materials, Technical University of Denmark, Lyngby, Denmark \\ ${ }^{\diamond}$ Wireless Networking and Communications Group, ECE Dept., University of Texas at Austin, Texas, US \\ Email: ${ }^{\dagger}\{$ huw, vbi $\} @$ com.dtu.dk, $\diamond$ jandrews@ece.utexas.edu
}

\begin{abstract}
This paper analyzes the potential uplink capacity gains of a two-hop 802.16j relay network operating in transparent mode. Detailed channel models and adaptive modulation and coding (AMC) in the physical layer are developed. To mitigate the capacity loss due to multihop communications, multi-input multi-output (MIMO) transmission is applied in the relay link. We derived the cell coverage and average spectral efficiency, as well as the system Erlang capacity supporting both voice and data traffic. The numerical results show that MIMO transmission in the relay link can significantly improve the end-to-end spectral efficiency. Furthermore, the position and the number of relay stations (RSs) have a great impact on the capacity gain. These results are further verified in the evaluation of the system Erlang capacity. The study demonstrates that with proper deployment of RSs and use of MIMO transmission in the relay link, significant capacity gains can be achieved with a two-hop 802.16 j relay system compared to the conventional single-hop 802.16e system.
\end{abstract}

\section{INTRODUCTION}

Initial field trial of WiMAX products showed that 802.16e system has limited coverage and provides unsatisfied qualityof-service (QoS) for indoor users as well as users at cell boundaries. To address this problem, the 802.16j task group was formed to develop a relay based solution to support mobile multihop relay (MMR) operation. In an MMR network, mobile stations (MSs) are allowed to route through intermediate relay stations (RSs) to reach the base station (BS), which differs from the legacy single-hop 802.16e system. A key advantage of MMR is that it can reduce installation and operation costs compared with deploying additional BSs to cover these areas, particularly in the early stage of network deployments. With the introduction of $\mathrm{RS}$, it becomes possible to break up a long single-hop link between a BS and a distant MS into a series of shorter hops. As the shorter hops are more spectrally efficient, the overall end-to-end spectral efficiency of MMR may increase relative to the single-hop system. RS may also be used as an attachment point for those MSs that are out of the communication range with the $\mathrm{BS}$, thereby extending cellular range. The $802.16 \mathrm{j}$ RS is fully backward compatible with an $802.16 \mathrm{e}$ MS, meaning that an MS can be supported by an $802.16 j$ relay network without any change.

Although the performance of multihop relay networks has been extensively studied, there have been a few studies concentrated on 802.16j MMR networks. Cell coverage and capacity are analyzed in [1] for a two-hop extension of an 802.16 cell in the presence of mesh communications. The results show the trade-off between coverage extension and capacity decrease. The capacity gain attainable with a relayenhanced $802.16 \mathrm{j}$ system is investigated in [2] via simulations. The results show that uplink capacity for both VoIP and data traffic increases significantly when RSs are deployed in the system. References [3] \& [4] compared network coverage and system capacity for 802.16j MMR networks with single-hop, transparent relay, and non-transparent relay with centralized and distributed scheduling mode. In [5], various cooperative diversity schemes for a two-hop relay network using the 802.16j standard are investigated. However, no previous work has developed specific physical layer models to analyze the potential capacity gains of $802.16 \mathrm{j}$ systems.

The objective of this work is to answer some initial questions regarding the capacity gains that can be achieved through the use of transparent relays. The study has two key aspects. First, we derive the average end-to-end cell spectral efficiency and analyze the impact of the location and the number of RSs on the capacity gains. Second, the Erlang capacity of the system supporting both voice and data traffic at a given QoS requirement is calculated and compared with/without relays.

The rest of the paper is organized as follows. In Section II, we introduce the system architecture as well as the frame structure of $802.16 \mathrm{j}$ MMR networks with transparent mode. In Section III, analytical models for calculating the average cell spectral efficiency as well as the system Erlang capacity are presented. Numerical results are discussed in Section IV. Finally, some conclusions are drawn in Section V.

\section{SYSTEM MODEL}

We consider a single cell $802.16 \mathrm{j}$ based cellular network. Two different relay modes are defined: transparent and nontransparent [4]. The key difference between these two modes of operation lies in the transmission of framing information. In transparent mode, the multihop relay base station (MRBS) transmits such information, while in non-transparent mode this information is transmitted by the RS. This leads to different benefits depending on the relay operation mode. More specifically, the transparent relays (T-RSs) do not extend cell coverage but aim to enhance the spectral efficiency within the MR-BS cell. The non-transparent relays (NT-RSs), on the other hand, provide coverage extension while the transmission of the framing information results in significant interference among the relays and therefore, limits the potential spectral efficiency enhancement. At present, $802.16 \mathrm{j}$ systems operate 

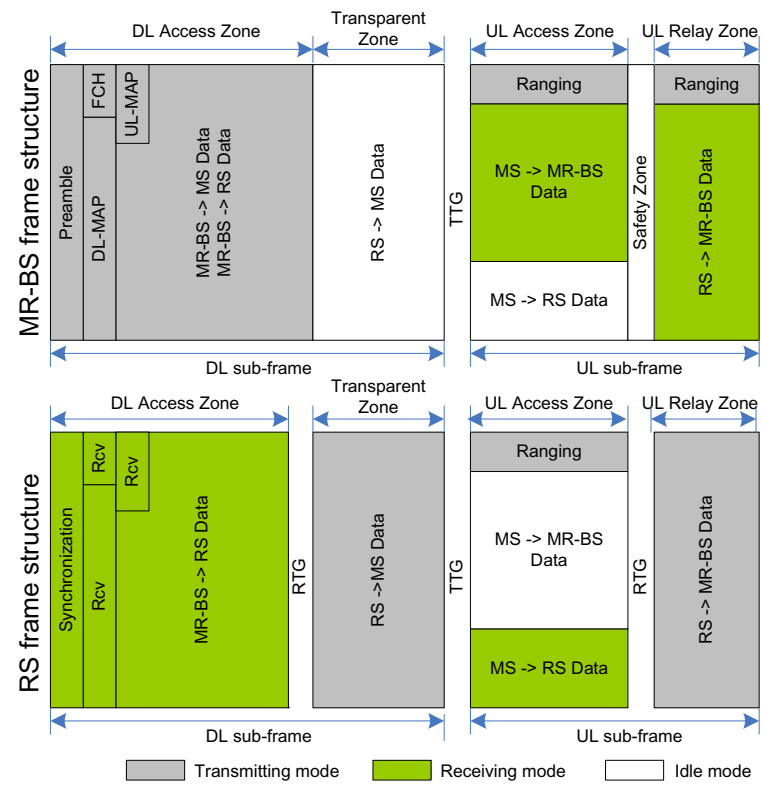

Fig. 1. Frame structure for a two-hop transparent relay system [3]

exclusively in either transparent or non-transparent mode. Thus, the deployment of RSs either enhances the overall spectral efficiency within the MR-BS cell coverage or increases the MR-BS cell coverage, but not both.

In this paper, we only consider transparent mode. A high level frame structure of $802.16 \mathrm{j}$ systems operating in transparent mode is shown in Fig. 1. The frame structure supports a typical two-hop relay-enhanced communication, where some MSs communicate directly to the MR-BS, while other MSs are attached to a T-RS and communicate to the MR-BS via the T-RS. Frame sections in green denote receive operation, sections in grey denote transmit operation, whereas sections in white denote idle mode. Even though extensions of the frame structure to support more than two hops have been defined by the work group, here we only concentrate on a two-hop relay-enhanced 802.16j MMR network, as the communication path between the MS and MR-BS is usually no more than two hops away in practical scenarios.

At the physical layer, the time axis is divided into frames with fixed length, each of which consists of a downlink (DL) and an uplink (UL) subframe to support TDD operation. In order to enable multihop communication, both downlink and uplink subframes are further divided into different transmission zones: one access zone and one relay zone. The access zone is dedicated for communication that directly engages MSs, and it is backward compatible with the 802.16e standard. Specifically, MSs receive from or transmit to the MR-BS or T-RS with which they are associated with in the access zone of downlink and uplink subframe, respectively. The access zone in both downlink and uplink is followed by a relay zone. In the relay zone, the T-RS receives from or transmits to the MR-BS in the corresponding downlink or uplink subframe, respectively. For legacy MSs, the notion of relay zone remains transparent to them, as they will only become aware of the existence of some new zones following the access zone based upon the DL-MAP and UL-MAP, and thus simply stay idle during these relay zones. In an MMR network, the radio link between the MS and their superordinate T-RS or MR-BS is defined as the access link, while the radio link between the T-RS and MR-BS is defined as the relay link.

In the access zone, adaptive modulation and coding (AMC) is employed at the physical layer for the MSs to adjust the data rate and transmission power dynamically according to the time-varying channel conditions. The transmitter only tracks and adapts to shadowing, which changes more slowly. In the relay zone, multiple transmit and receive antennas are employed at the MR-BS and T-RSs to increase the transmission capacity over the relay link.

In this paper, we only focus on the uplink capacity of a twohop relay-enhanced $802.16 \mathrm{j}$ system operating in transparent mode. Two types of services, voice and data are supported by the system. Voice is not tolerant to packet delay, thus requires a constant bit rate. Data is more elastic in the transmission rate regarding the channel conditions, but also requires a minimum throughput. Voice and data users arrive at the cell randomly.

\section{AnAlytical Models}

In this section, we first derive the average cell spectral efficiency under detailed channel models. Then we use the classical teletraffic theory to calculate the Erlang capacity of the system supporting both voice and data traffic.

\section{A. Channel Models}

Assume that the channel has quasi-static flat fading. We use the following notation:

$\overline{\bar{\gamma}}$ denotes the average SNR per symbol for a fixed path loss, averaged over both shadowing and fast fading.

$\bar{\gamma}$ denotes the random SNR per symbol for a fixed path loss and shadowing. Its average value, averaged over shadowing, is $\overline{\bar{\gamma}}$.

$\gamma$ denotes the random SNR per symbol for a fixed path loss, shadowing, and fast fading. Its average value, averaged over fast fading, is $\bar{\gamma}$.

The channel model consists of three parts: path loss, slow fading, and fast fading [7].

1) Path-Loss Model: Assume a node transmits at power $P_{t}$ over a distance $r$. The received SNR over path loss is then $\overline{\bar{\gamma}}(r)=P_{t} K\left(\frac{r_{0}}{r}\right)^{\alpha} /\left(N_{0} B\right)$, where $\alpha$ is the path loss exponent, $K=\left[\lambda /\left(4 \pi r_{0}\right)\right]^{2}$ is a unitless constant that depends on the wavelength of the RF carrier $\lambda$ and outdoor reference distance $r_{0}$, and $N_{0} B$ is the thermal noise power.

2) Slow Fading (Shadowing): With shadowing, the received SNR can be expressed as $\bar{\gamma}(r)=P_{t} K\left(\frac{r_{0}}{r}\right)^{\alpha} \chi /\left(N_{0} B\right)$, where the shadowing value $\chi$ is typically modeled as a log-normal random variable $10 \log _{10} \chi \sim \mathcal{N}\left(0, \sigma_{\mathrm{dB}}^{2}\right)$.

3) Fast Fading: In Rayleigh fading channel, the received signal amplitude $z$ has the Rayleigh distribution $p(z)=$ $\frac{z}{\sigma^{2}} e^{-z^{2} / 2 \sigma^{2}}$, and the received SNR is exponentially distributed $p_{\gamma}(\gamma \mid \bar{\gamma})=\frac{1}{\bar{\gamma}} e^{-\gamma / \bar{\gamma}}$ with mean $\bar{\gamma}$. 


\section{B. Adaptive Rate and Power Allocation in Access Zone}

The objective of AMC is to maximize spectral efficiency by adjusting the transmission parameters according to timevarying channel conditions, while maintaining a prescribed bit error rate (BER) over wireless links. In this paper, we examine adaptive schemes in terms of continuous power and discrete rate adaptation for composite fading channels consisting of both slow (shadowing) and fast fading. Since the fast fading changes too quickly to be accurately tracked, the transmitter only adapts to the slow fading.

Let $N$ denote the total number of transmission modes available and $\mathcal{M}=\left\{M_{0}, \ldots, M_{N-1}\right\}$ denote the set of constellations, where $M_{0}=0$ corresponds to no data transmission. The rate $K(\bar{\gamma})$ associated with each transmission mode is $K_{j}=\log _{2} M_{j}(j=0, \ldots, N-1)$. We partition the entire $\bar{\gamma}$ range into $N$ non-overlapping consecutive intervals with boundaries denoted as $\Gamma_{j}(j=-1, \ldots, N-1)$, where $\Gamma_{-1}=0$ and $\Gamma_{N-1}=\infty$. Each rate $K_{j}$ is assigned to a fading region when $\bar{\gamma} \in\left[\Gamma_{j-1}, \Gamma_{j}\right)$. We want to optimize the boundaries $\Gamma_{j}(j=0, \ldots, N-2)$ and find the optimal power $P(\bar{\gamma})$ and rate $K(\bar{\gamma})$ adaptation that maximize the average rate $\mathbf{E}[K(\bar{\gamma})]$ with average transmit power $\bar{P}$, while meeting a given BER target $\bar{P}_{b}$.

Assuming Rayleigh fading for the fast fading, the average bit error probability for a given shadowing $\bar{\gamma}$ can be approximated by averaging the instantaneous bit error probability over the fast fading distribution [6]:

$$
\bar{P}_{b}(\bar{\gamma})=\frac{0.2}{\frac{1.5 \bar{\gamma} P(\bar{\gamma}) / \bar{P}}{2^{K(\bar{\gamma})}-1}+1}
$$

Rearranging Eqn. (1) yields the power adaptation scheme:

$$
\frac{P(\bar{\gamma})}{\bar{P}}=\frac{h[K(\bar{\gamma})]}{\bar{\gamma}}=\frac{h\left[K_{j}\right]}{\bar{\gamma}}, \quad \Gamma_{j-1} \leq \bar{\gamma}<\Gamma_{j}
$$

where

$$
h[K(\bar{\gamma})]=\frac{\frac{0.2}{\bar{P}_{b}}-1}{1.5}\left(2^{K(\bar{\gamma})}-1\right)
$$

We maximize spectral efficiency by maximizing:

$$
\mathbf{E}[K(\bar{\gamma})]=\sum_{j=0}^{N-1}\left[K_{j} \int_{\Gamma_{j-1}}^{\Gamma_{j}} p(\bar{\gamma}) d \bar{\gamma}\right]
$$

subject to the power constraint:

$$
\int_{0}^{\infty} \frac{P(\bar{\gamma})}{\bar{P}} p(\bar{\gamma}) d \bar{\gamma}=\sum_{j=0}^{N-1} \int_{\Gamma_{j-1}}^{\Gamma_{j}} \frac{h\left[K_{j}\right]}{\bar{\gamma}} p(\bar{\gamma}) d \bar{\gamma}=1
$$

where $p(\bar{\gamma})$ is the pdf of shadowing $\bar{\gamma}$.

The optimal region boundaries $\Gamma_{j}(j=0, \ldots, N-2)$ can be obtained by using the Lagrange equation [6].

\section{MIMO Transmission in Relay Zone}

Generally it is expected that the relay link enjoys better channel quality than the access link, as it is likely that there is line-of-sight (LOS) communication between T-RSs and MRBS. In this case, the received signal amplitude $z$ over relay link follows the Rician distribution.
In UL relay zone, the T-RS relays the traffic received from the MSs to the MR-BS. To satisfy the flow conservation constraint, the capacity of relay link should be high enough to accommodate all the traffic received by the T-RS in the UL access zone. Multi-input multi-output (MIMO) scheme is an emerging technology that can be used to increase data rates through multiplexing or to improve the transmission reliability through diversity without requiring increased signal power or bandwidth. In 802.16j MMR networks, the capacity of relay link has a great impact on the overall end-to-end spectral efficiency, thus MIMO transmission with multiplexing scheme is applied to increase the link capacity.

Let $M_{t}$ and $M_{r}$ denote the number of transmit and receive antennas, respectively. Consider a time-varying MIMO channel with $M_{r} \times M_{t}$ channel gain matrix $\mathbf{H}$. We assume that the receiver has perfect channel state information (CSI), but the transmitter does not. The transmitter assumes a zero-mean spatially white (ZMSW) distribution for $\mathbf{H}$.

A reliable communication rate with CSI at receiver in fading channels can be expressed as [6]:

$$
K_{C}=\max _{\mathbf{R}_{\mathbf{x}}: \operatorname{Tr}\left(\mathbf{R}_{\mathbf{x}}\right) \leq \bar{P}} \mathbf{E}_{\mathbf{H}}\left[\log _{2} \operatorname{det}\left(\mathbf{I}_{M_{r}}+\frac{g}{\sigma^{2}} \mathbf{H R}_{\mathbf{x}} \mathbf{H}^{H}\right)\right]
$$

where $g$ is the attenuation function due to path loss, $\mathbf{R}_{\mathbf{x}}$ is the input covariance matrix, $\operatorname{Tr}\left(\mathbf{R}_{\mathbf{x}}\right)$ is the trace of $\mathbf{R}_{\mathbf{x}}$ which corresponds to the total transmit power constraint. The optimum input covariance matrix that maximizes rate $K_{C}$ for the ZMSW model is the scaled identity matrix $\mathbf{R}_{\mathbf{x}}=$ $\left(\bar{P} / M_{t}\right) \mathbf{I}_{M_{t}}$, that is, the transmit power is equally distributed among all the transmit antennas. Then (5) can be rewritten as:

$$
\begin{aligned}
K_{C} & =\mathbf{E}_{\mathbf{H}}\left[\log _{2} \operatorname{det}\left(\mathbf{I}_{M_{r}}+\frac{\bar{P} g}{\sigma^{2} M_{t}} \mathbf{H H}^{H}\right)\right] \\
& =\sum_{i=1}^{R_{\mathbf{H}}} \mathbf{E}_{\mathbf{H}}\left[\log _{2}\left(1+\frac{\bar{P} g \lambda_{i}}{\sigma^{2} M_{t}}\right)\right]
\end{aligned}
$$

where $R_{\mathbf{H}}$ denotes the rank of $\mathbf{H}$, and $\lambda_{i}$ is the $i$ th largest eigenvalue of $\mathbf{H H}^{H}$

\section{Relay Selection Strategy and Cell Spectral Efficiency}

With two-hop relaying, the transmission requires two phases. In the first phase source-to-relay communication takes place and the second phase is used for the relays to forward the received information to the destination. The two-phase transmission causes multiplexing loss since each data block has to be transmitted twice, over both the access link and the relay link. Hence relay must be used only when it can provide better end-to-end spectral efficiency $\rho$ (in bits/s/Hz) than that of direct transmission, i.e., without relay.

Let's consider a MS located at $(r, \theta)$ using polar coordinates, where $r$ and $\theta$ are the distance and the angle to the MRBS respectively. We assume that the T-RSs are symmetrically positioned in the cell with the same distance to the MR-BS, thus the spectral efficiency of each relay link $K_{C}$ can be assumed identical using (6). When the MS transmits to the MR-BS directly, its spectral efficiency is $\rho_{\mathrm{D}}(r, \theta)=\mathbf{E}\left[K\left(\bar{\gamma}_{\mathrm{D}}\right)\right]$ 
using (3), where $\bar{\gamma}_{\mathrm{D}}$ is the received SNR at the MR-BS under path loss and shadowing. When the MS uses relay, its endto-end spectral efficiency including the effect of multiplexing loss can be expressed as:

$$
\rho_{\mathrm{R}}(r, \theta)=\frac{\mathbf{E}\left[K\left(\bar{\gamma}_{\mathrm{R}}\right)\right] K_{C}}{\mathbf{E}\left[K\left(\bar{\gamma}_{\mathrm{R}}\right)\right]+K_{C}}
$$

where $\bar{\gamma}_{\mathrm{R}}$ is the received SNR at the closet T-RS under path loss and shadowing. The end-to-end spectral efficiency from a MS located at $(r, \theta)$ to the MR-BS is then given by:

$$
\rho(r, \theta)=\max \left\{\rho_{\mathrm{D}}(r, \theta), \rho_{\mathrm{R}}(r, \theta)\right\}
$$

The average cell spectral efficiency can be obtained by integrating the end-to-end spectral efficiency over the cell area:

$$
\bar{\rho}=\frac{1}{\pi R^{2}} \int_{0}^{2 \pi} \int_{0}^{R} \rho(r, \theta) r d r d \theta
$$

\section{E. Erlang Capacity of the System}

The Erlang capacity is generally defined as the maximum traffic load that the system can support when the blocking probabilities at the call admission control (CAC) level do not exceed certain thresholds. The Erlang capacity of the system can be obtained by using the classical teletraffic model of multi-dimensional loss systems [8].

We assume that voice and data users arrive at the cell in a random order following a Poisson process with rate $\lambda_{v}$ and $\lambda_{d}$, respectively. The service time for voice and data users is exponentially distributed with intensity $\mu_{v}$ and $\mu_{d}$, respectively. The CAC module decides whether an incoming call should be admitted or not. To prioritize voice service, CAC reserves a fixed amount of bandwidth dedicated for voice users only, and allows both voice and data users to compete for the remaining bandwidths.

For a given quality of service, voice users require a constant bit rate of $R_{v}$ bits per second and data users require a minimum throughput of $R_{d}$ bits per second. Let $B$ denote the total amount of bandwidth available for UL data transmissions, $\Delta B$ of them are reserved for voice users only. Given the average cell spectral efficiency, each voice user is allocated $\bar{c}_{v}=R_{v} / \bar{\rho}$ bandwidth on average to ensure a constant bit rate, and each data user is allocated at least $\bar{c}_{d}=R_{d} / \bar{\rho}$ bandwidth on average to guarantee a minimum throughput. The remaining bandwidths can be allocated to data users on demand to increase their throughput. Let $\left\{n_{v}, n_{d}\right\}$ denote the number of admitted voice users and data users in the cell, respectively. Under the above conditions, the state space of the system is given by:

$$
S:=\left\{n_{v}, n_{d}\right\} \in M \times N
$$

subject to:

$$
\begin{gathered}
n_{v} \cdot \bar{c}_{v}+n_{d} \cdot \bar{c}_{d} \leq B \\
n_{d} \cdot \bar{c}_{d} \leq B-\Delta B
\end{gathered}
$$

where $M$ and $N$ are the maximum number of voice and data users that can be accommodated in the system, respectively.
The call-level characteristics of a multi-class (voice/data) 802.16j MMR network with the CAC policy described above can be modeled by a Continuous Time Markov Chain (CTMC), shown in Figure 2, where state $(i, j)$ represents the amount of bandwidth occupied by voice users and data users, respectively. As it is a reversible Markov process (Kolmogorov's criteria is fulfilled for all possible paths) and has product form, the numerical evaluations can be done by using the convolution algorithm [8].

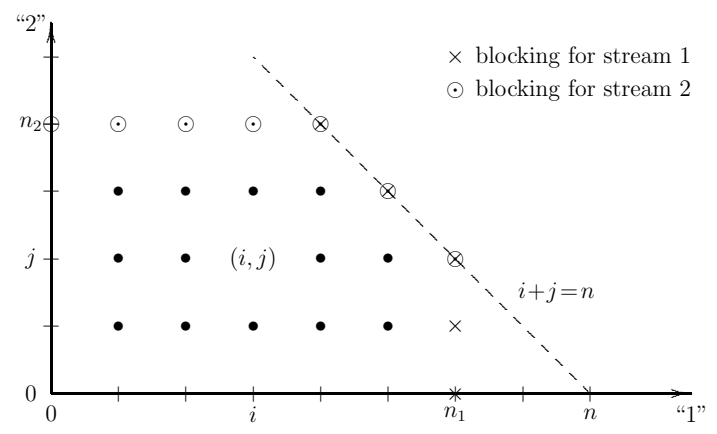

Fig. 2. Structure of the state transition diagram for two-dimensional traffic processes with class limitations. When calculating the equilibrium probabilities, state $(i, j)$ can be expressed by state $(i, j-1)$ and recursively by state $(i, 0),(i-1,0)$, and finally by $(0,0)$. [8]

Let $p_{v}(\cdot)$ and $p_{d}(\cdot)$ denote the state probabilities of each service class as if it is alone in the system. By applying Erlang B-formula, we get:

$$
\begin{aligned}
& \begin{cases}p_{v}\left(m \cdot \bar{c}_{v}\right)=\frac{\frac{A_{v}^{m}}{m !}}{\sum_{i \in \Omega_{v}} \frac{A_{v}^{i}}{i !}} & m \in \Omega_{v} \\
p_{v}(\cdot)=0 & \text { else }\end{cases} \\
& \begin{cases}p_{d}\left(n \cdot \bar{c}_{d}\right)=\frac{\frac{A_{d}^{n}}{n !}}{\sum_{j \in \Omega_{d}} \frac{A_{d}^{j}}{j !}} & n \in \Omega_{d} \\
p_{d}(\cdot)=0 & \text { else }\end{cases}
\end{aligned}
$$

where $A_{v}=\lambda_{v} / \mu_{v}$ and $A_{d}=\lambda_{d} / \mu_{d}$ are the offered traffic of voice and data users, respectively. The offered traffic is usually defined as the average number of call attempts per mean holding time. $\Omega_{v}$ and $\Omega_{d}$ are the sets of possible number of voice and data users in each service class, $\lfloor x\rfloor$ denotes the largest integer not exceeding $x$.

$$
\begin{aligned}
& \Omega_{v}:=\left\{0,1, \ldots,\left\lfloor\frac{B}{\bar{c}_{v}}\right\rfloor\right\} \\
& \Omega_{d}:=\left\{0,1, \ldots,\left\lfloor\frac{B-\Delta B}{\bar{c}_{d}}\right\rfloor\right\}
\end{aligned}
$$

The closed-form of the call blocking probability of each traffic stream can be obtained by using the convolution algorithm, shown in (13) and (14). Due to the PASTA-property in Poisson arrival process, the call congestion is also equal to the time congestion and traffic congestion.

\section{NumERICAL RESUlts AND Discussions}

We consider a single-cell two-hop 802.16j MMR network operating in transparent mode with a total bandwidth of 5 


$$
\begin{gathered}
\mathrm{P}_{\text {block }}^{v}=\frac{\sum_{i=\left\lfloor\frac{B}{\bar{c}_{v}}\right\rfloor}^{\left\lfloor\frac{B}{\bar{c}_{v}}\right\rfloor}\left\{\lambda_{v} \cdot p_{v}\left(i \cdot \bar{c}_{v}\right) \cdot p_{d}\left(\left\lfloor\frac{B-i \cdot \bar{c}_{v}}{\bar{c}_{d}}\right\rfloor \cdot \bar{c}_{d}\right)\right\}}{\sum_{i=0}^{\left\lfloor\frac{\Delta B}{\bar{c}_{v}}\right\rfloor}\left\{\lambda_{v} \cdot p_{v}\left(i \cdot \bar{c}_{v}\right) \cdot \sum_{j=0}^{B-\Delta B} p_{d}(j)\right\}+\sum_{i=\left\lfloor\frac{B}{\bar{c}_{v}}\right\rfloor}^{\left.\frac{B}{\bar{c}_{v}}\right\rfloor+1}\left\{\lambda_{v} \cdot p_{v}\left(i \cdot \bar{c}_{v}\right) \cdot \sum_{j=0}^{B-i \cdot \bar{c}_{v}} p_{d}(j)\right\}} \\
\mathrm{P}_{\text {block }}^{d}=\frac{\sum_{i=0}^{\left\lfloor\frac{B-\Delta B}{\bar{c}_{d}}\right\rfloor-1}\left\{\lambda_{d} \cdot p_{d}\left(i \cdot \bar{c}_{d}\right) \cdot p_{v}\left(\left\lfloor\frac{\left.B-i \cdot \bar{c}_{d}\right\rfloor}{\bar{c}_{v}}\right\rfloor \cdot \bar{c}_{v}\right)\right\}+\lambda_{d} \cdot p_{d}\left(\left\lfloor\frac{B-\Delta B}{\bar{c}_{d}}\right\rfloor \cdot \bar{c}_{d}\right) \cdot \sum_{j=0}^{B-\left\lfloor\frac{B-\Delta B}{\bar{c}_{d}}\right\rfloor \cdot \bar{c}_{d}} p_{v}(j)}{\sum_{i=0}^{\left\lfloor\frac{B-\Delta \Delta B}{\bar{c}_{d}}\right\rfloor}\left\{\lambda_{d} \cdot p_{d}\left(i \cdot \bar{c}_{d}\right) \cdot \sum_{j=0}^{B-i \cdot \bar{c}_{d}} p_{v}(j)\right\}}
\end{gathered}
$$

$\mathrm{MHz}$, where the carrier frequency is $2.5 \mathrm{GHz}$. The system is configured such that $50 \%$ of the total capacity is used for downlink and uplink transmissions, respectively. Assume that $10 \%$ of the uplink bandwidth is reserved for voice traffic only. The signalling overhead in PHY and MAC layers is not considered for simplicity. For the access link, the channel model follows a path-loss exponent of 3.5, a log-normal shadowing with mean 0 and variance $4 \mathrm{~dB}$, and Rayleigh fading. For the relay link, the channel model follows a pathloss exponent of 3 and Rician fading with factor $K$ of 10 . Each MR-BS and T-RS is equipped with multiple transmit and receive antennas. The relay link is modeled as a MIMO channel with perfect CSIR. The transmission power of the MR-BS and T-RS is fixed at $1 \mathrm{~W}$ and $0.5 \mathrm{~W}$, respectively, while the average transmission power of the MS is $100 \mathrm{~mW}$. The modulation order and coding rate in AMC is determined by the received SNR $\bar{\gamma}$ under path loss and shadowing. We follow the AMC table shown in Table I, which specifies the minimum SNR $\bar{\gamma}$ required to meet a target average BER, e.g., $10^{-4}$. We set the target outage probability of the cell to be $5 \%$, which is usually considered to be an acceptable QoS requirement. The cell radius under these constraints can be obtained in [6], which is close to $2 \mathrm{~km}$.

\begin{tabular}{|c|c|c|c|}
\hline $\begin{array}{c}\text { Modulation } \\
\text { scheme }\end{array}$ & $\begin{array}{c}\text { Coding } \\
\text { rate }\end{array}$ & bits/symbol & $\begin{array}{c}\text { Minimum } \bar{\gamma} \text { for } \\
10^{-4} \text { BER }(\mathbf{d B})\end{array}$ \\
\hline QPSK & $1 / 2$ & 1 & 3.5 \\
\hline QPSK & $3 / 4$ & 1.5 & 6.5 \\
\hline 16QAM & $1 / 2$ & 2 & 9.5 \\
\hline 16QAM & $3 / 4$ & 3 & 12.5 \\
\hline 64QAM & $2 / 3$ & 4 & 16.5 \\
\hline 64QAM & $3 / 4$ & 4.5 & 18.5 \\
\hline
\end{tabular}

TABLE I

ADAPTIVE MODULATION AND CODING SCHEMES FOR 802.16

We assume that the T-RSs are placed symmetrically in the cell with the same distance to the MR-BS. We also assume that the MSs are uniformly distributed in the cell. The constant bit rate requirement for voice users is set to be $50 \mathrm{Kbits} / \mathrm{sec}$, and the minimum throughput constraint for data users is set to be $100 \mathrm{Kbits} / \mathrm{sec}$.

To compare the spectral efficiency with direct transmissions and with relays, we define the spectral efficiency gain $G$ at cell location $(r, \theta)$ in polar coordinates as:

$$
G(r, \theta)=\frac{\rho(r, \theta)-\rho_{D}(r, \theta)}{\rho_{D}(r, \theta)} \times 100 \%
$$

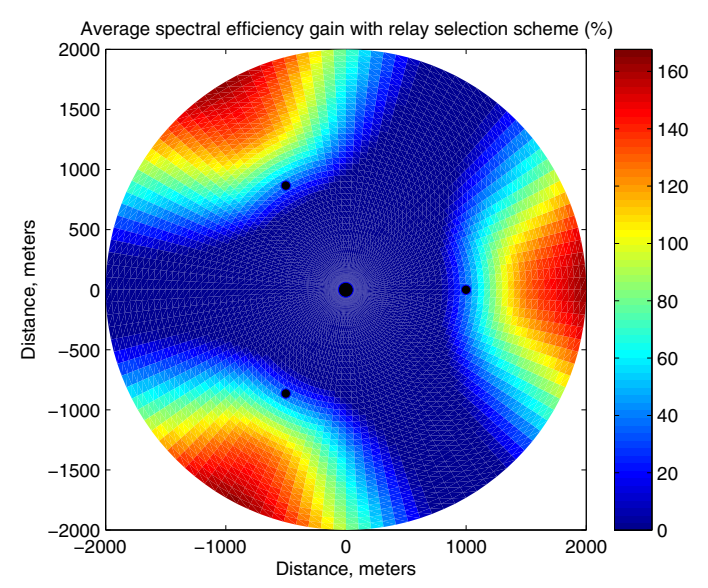

Fig. 3. Spectral efficiency gain with 3 T-RSs deployed per cell at a distance of $1 \mathrm{~km}$ away from the MR-BS, $4 \times 4$ MIMO in relay link, compared with direct transmissions

where $\rho_{D}(r, \theta)$ and $\rho(r, \theta)$ denote the end-to-end spectral efficiency with direct transmissions and with relay selection strategy discussed in Section III-D, respectively.

Fig. 3 shows the spectral efficiency gain with three T-RSs deployed in the cell and $4 \times 4 \mathrm{MIMO}$, compared with direct transmissions. We observe that at the cell edge, where the distance is close to the T-RS but far away from the MR-BS, the spectral efficiency gain is significant and can achieve up to $160 \%$ compared to direct transmissions. In the region where the distance to the MR-BS is comparable to the closest T-RS, the gain is almost zero. This can be explained as follows. Since the relay link enjoys better channel quality and a $4 \times 4$ MIMO transmission is applied, the spectral efficiency over the relay link is much higher than that over the access link. Therefore the overall end-to-end spectral efficiency is mainly limited by the channel capacity of the access link, which further depends on the distance from a MS to the closest T-RS or MR-BS.

Fig. 4 depicts the impact of different T-RSs locations as well as different number of T-RSs on the average cell spectral efficiency. The deployment of T-RSs always exhibits better performance than direct transmissions, and the spectral efficiency increases as the number of T-RSs increases. This is in accordance with our expectations, as the increase of the number of T-RSs will increase the beneficial area in a cell where direct communication with MR-BS experiences unsatisfactory level of channel quality due to high path loss. 


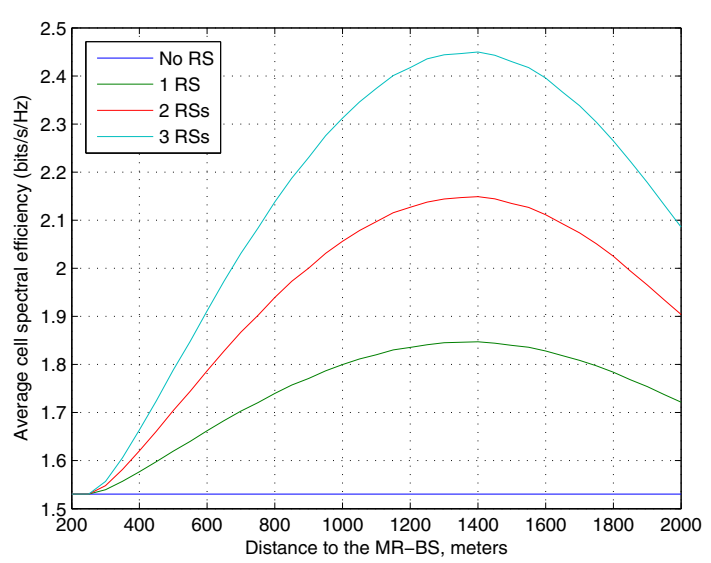

Fig. 4. Average cell spectral efficiency with $4 \times 4$ MIMO applied in relay link, over different T-RSs locations and with different number of T-RSs

Furthermore, we can see that the spectral efficiency increases as the distance from a T-RS to the MR-BS increases until it reaches a peak point. Then the spectral efficiency decreases as the distance increases. Let's revisit Fig. 3 and imagine that the T-RSs are moving away from the center. When the T-RSs are close to the MR-BS, the received SNR at MR-BS and T-RS is comparably the same, thus there is little gain by relaying. As the relays move away from the MR-BS, the region where the distance to the closest T-RS is much shorter than to the MRBS increases until the relays reach certain positions (around $1.2 \mathrm{~km}$ to the MR-BS), where the beneficial area by relaying is maximized. After that point, the beneficial area shrinks and hence, the average spectral efficiency decreases.

A two dimensional Erlang capacity region of voice and data traffic at $3 \%$ blocking probability is depicted in Fig. 5. The solid lines indicate the Erlang capacity region of voice traffic and the dashed lines indicate the Erlang capacity region of data traffic. The 'no RS' curve corresponds to the performance of conventional single-hop 802.16e network, while the remaining curves characterize the performance of a two-hop $802.16 \mathrm{j}$ MMR network. The figure shows that significant higher traffic loads can be accommodated by using relays, and the Erlang capacity region of both voice and data traffic increases as the number of T-RSs deployed per cell increases. This is due to the reason that when the number of T-RSs deployed per cell increases, more users that are far from the MR-BS will be served by the T-RSs with higher spectrum efficiency, therefore significantly increase the average cell spectral efficiency, which results in an enlargement of the system Erlang capacity region. Furthermore, we notice that the Erlang capacity region of voice traffic is larger than that of data traffic. This is because data service has a higher bit rate requirement than voice service, thus occupies more bandwidth than voice service does. We also notice that the system can always accommodate certain amount of voice traffic regardless of the load of data traffic, this is due to the bandwidth reservation policy applied to the voice traffic.

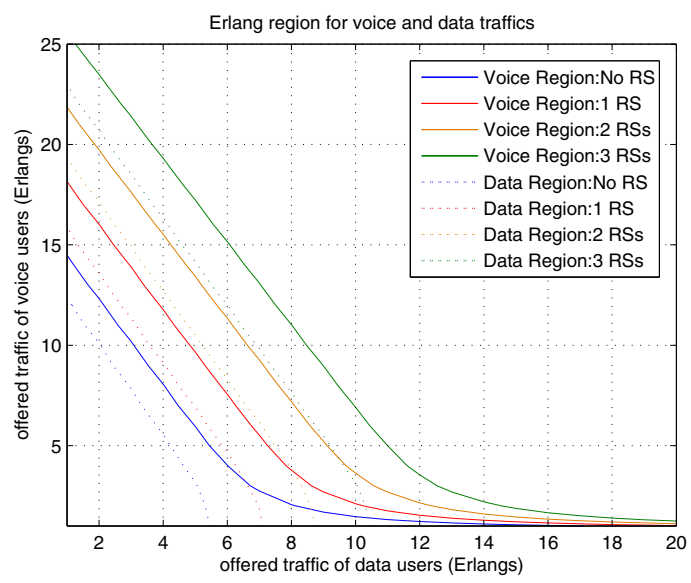

Fig. 5. Two dimensional Erlang capacity region of voice and data traffic with different number of T-RSs deployed per cell at a distance of $1 \mathrm{~km}$ away from the MR-BS, $4 \times 4$ MIMO in relay link, target blocking probability $3 \%$

\section{CONCLUSIONS}

In this paper, we have investigated the uplink capacity gain attainable with a two-hop 802.16j MMR network operating in transparent mode. To increase the spectral efficiency, AMC and MIMO transmission are applied in the access zone and relay zone, respectively. We have derived the cell coverage as well as the average spectral efficiency with direct transmission and with relay selection strategy. Also we have calculated the Erlang capacity of the system supporting both voice and data traffic. Numerical results show that with the introduction of relay stations and MIMO transmission applied in the relay link, the spectral efficiency of a two-hop relay system can be significantly increased compared with the single-hop system. However, the position and the number of T-RSs have a great impact on the capacity gain. With proper deployment of relays, significant capacity gains can be achieved.

\section{REFERENCES}

[1] G. S. Paschos, P. Mannersalo, and T. M. Bohnert, "Cell Capacity for IEEE 802.16 Coverage Extension", 5th IEEE Consumer Communications and Networking Conference, pp. 933-937, 2008.

[2] V. Eugene, B. Junjik, P. Roger, B. Randall, and L. Michael, "On the Uplink Capacity of an 801.16j System", IEEE Wireless Communications and Networking Conference, pp. 2657-2662, 2008

[3] H. Zeng, and C. Zhu, "System-Level Modeling and Performance Evaluation of Multi-Hop 802.16j Systems", International Wireless Communications and Mobile Computing Conference, pp. 354-359, 2008.

[4] V. Genc, S. Murphy, and J. Murphy, "Performance analysis of transparent relays in 802.16j MMR networks", 6th International Symposium on Modeling and Optimization in Mobile, Ad Hoc, and Wireless Networks and Workshops, pp. 273-281, 2008.

[5] B. Can, H. Yanikomeroglu, F. A. Onat, E. D. Carvalho, and H. Yomo, "Efficient Cooperative Diversity Schemes and Radio Resource Allocation for IEEE 802.16j", IEEE Wireless Communications and Networking Conference, pp. 36-41, 2008.

[6] A. J. Goldsmith, Wireless Communications, Cambridge University Press, 2005.

[7] J. G. Andrews, A. Ghosh, and R. Muhamed, Fundamentals of WiMAX, Prentice Hall, 2007.

[8] V. B. Iversen, Teletraffic Engineering Handbook, COM department, Technical University of Denmark, 2005. 